\title{
Interactive cross-detector analysis of vortical flow data
}

\author{
Raphael Bürger ${ }^{1,2}$, Philipp Muigg 2 , Helmut Doleisch ${ }^{2}$, Helwig Hauser $^{2}$ \\ ${ }^{1}$ Institute of Computer Graphics, Vienna University of Technology, Austria \\ ${ }^{2}$ VRVis Research Center, Vienna, Austria
}

\begin{abstract}
In this paper we discuss the application of multiple linked views for advanced vortex investigation in flow data. Local feature detectors and additional measures integrate into an interactive flow feature detection system based on multiple linked views. We discuss how linking and brushing of derived feature information can lead to increased performance in interactive feature analysis. In a case study we demonstrate how the possibility to integrate the information of multiple detectors leads to sound understanding of vortex type features. Enhanced credibility and combined advantages of several detectors can be achieved by uniting the results of multiple feature detectors in multiple coordinated views.
\end{abstract}

\section{Introduction}

Flow is everywhere. Gaseous or liquid flows occur on all scales from capillary flows transporting oxygen to a muscle to the motion of plasma inside the sun. One fundamental property of flow is the ubiquitous nature of turbulence. A century ago many people believed there might be some kind of 'universal theory of turbulence' that was supposed to be valid under a wide range of circumstances. It is now generally agreed that such a theory does not exist [6]. Since there is no general theory that describes turbulent fluids many engineers do not fully trust automated feature extraction methods. Therefore, in the context of flow visualization, interactive approaches are an important tool to understand the complex movements inside the flow and the changes of related attributes such as temperature and pressure.

Computational fluid dynamics (CFD) methods output simulation data with ever increasing complexity. The engineer wants to answer questions like: What is the flow like now? How does the flow change over time? Where does the flow lead to? As long as the turbulence is not developed into chaos, there still exist coherent structures that allow predictions and can give insight in the behavior of the flow [11]. Vortices are an important structure and by now we know a lot on their behavior. It is widely agreed that vortices belong to the most important coherent features in flow fields. Therefore tools that can help to find and ana- lyze the parts of the fluid that exhibit swirling motion can greatly help the engineer or physicist to gain insight into the properties of the fluid motion.

There is no ultimate agreement on how to generally define and detect vortices. Even though the concept of a vortex is common in fluid dynamics and flow visualization has proven useful to describe and model the behavior of fluids. Therefore there exists a wide range of local and global feature detectors that can find vortices in the flow under the right conditions [19]. They have individual advantages and can outperform others in certain situations. Some of the intrinsic properties of a vortex detector include:

- Representation of the vortex: vortex core methods extract line type features giving precise insight into the location of the vortex core. Vortex region extraction algorithms extract an approximate volume that describes the extent of the connected region of swirling motion.

- Galilean invariance: a vortex detection algorithm that is Galilean invariant is valid in all inertial frames of reference [12]. A detector that has this property is able to find vortices also in cases where a steady current in one direction is added to the flow field and masks the vortical motion of the fluid.

- Robustness: many detection schemes are considered to be prone to errors in special configurations of the flow. Robustness can vary with respect to noise in the data and the size of the grid in unique ways for each detector [20].

In the remainder of this paper we will see how multiple linked views can give access to many of the useful properties of the different detectors at once, but in the scope of this paper we will limit the analysis to vortex region extraction based approaches. These are of interest, since vortices are especially difficult to detect and even approved extraction algorithms can differ in their outputs significantly.

While most detectors are prone to find false positives [9], because the higher order derivatives are subject to frequency amplification, they do not share exactly the 
same numerical issues. In this paper we discuss how the different vortex extraction schemes can interact and complement each other using interactive visual analysis.

This is also an example on how visual analysis can benefit from multiple views that allow the user to build a complex mental model of the properties of the flow. Until recently the rising computing power has led mainly to rising complexities in the data generated. We propose higherorder features for visual analysis as a tool to meet this challenge by incorporating complex automated feature detectors into the process of visual analysis.

The contribution of this paper is: the presentation of a general multi-view framework where smooth feature detectors are combined for interactive analysis. The adaption of the $P, Q$ and $R$ plots to an analytic view based on scatterplots. An extension of classical rectangular brushing to integrate visual analysis and automated feature detection based on multiple detectors. Furthermore we present analytical interaction schemes for interactive visual analysis based on multiple views that give a general approach to vortex type feature analysis.

\section{Related Work}

In this section we will shortly recapitulate related work in visual analysis of scientific datasets. We will give some background on the SimVis [28, 7] approach to visual data analysis, especially the concept of smooth brushing and linking. Then we will give an overview on the vortex detectors that we have implemented to combine them into a multiview vortex inspection framework.

Interactive extraction of information has been a hot research topic in recent years focusing on interactive information drill-down [24], visual data mining [29, 13] and visual analytics [26]. Important issues are advanced interaction concepts as well as procedures and algorithms to gain access to features and information in the data.

Recently developed visualization techniques and modern graphics hardware make it possible to interact with vast amounts of multidimensional data in an intuitive way. The SimVis system aims at the interactive visual analysis of large, multi-dimensional and time-dependent datasets from CFD simulation. To obtain a feature-based flow visualization, different forms of data visualization are combined for data exploration and analysis. Advanced interaction mechanisms enable the user to intuitively specify features in the flow data. Multiple, linked views [1] are used to show different aspects of the flow data. It is important that all views which concurrently show the same dataset are consistent among each other with respect to the selected features.

In Figure 1 we see an example for this approach. Multiple views and smooth brushing improve the flow feature analysis. The two 3D views show (a) regions of high local vortex-measure and (b) low velocity and high temperature in focus and serve as reference. (c) The scatterplot of a vortex detector $\left(\lambda_{2}\right)$ vs. its local extrema can be used to get an overall impression on the distribution of detector response values and brushing can be used to select vortical regions. In all attribute views the brushed elements appear red. Elements that are colored black are not selected in any of the views. Dots are colored green to show data items that are not selected in the current view but in other linked vies, i.e. they show the difference between the current selection from selections in other views. (d) The histogram shows the development of the features selected in the scatterplots (using color linking between views) and reveals two peaks of turbulence over time. This could be time steps that qualify for further investigation. (e) A scatterplot of velocity vs. temperature allows to compare attribute values of the selection (red) with those of the vortex regions (green).

Details on the vortex detectors and references will follow in Section 3. The combination of multiple classifiers has received much attention in the machine learning and and statistic communities. Lam [14] discuss methodologies for the combination of multiple classifiers to obtain improved recognition results. Roli and Kittler [21] present an overview on fusion methodologies.

\section{Detectors and flow measures}

In this section we give a short overview on the feature extraction techniques implemented in the context of this paper. A more general overview on flow feature extraction algorithms and visualization was published by Post et al. [19].

\subsection{Flow measures}

From engineers and from literature we have found a series of additional flow attributes to be useful for inspection and to understand the properties of vortices in the data set: Pressure $p$ can give hints to the location of a vortex. The swirling motion around the center of rotation can result in lower pressure in the vortex core region [17].

Vorticity (or curl) $\omega=\nabla \times \mathbf{v}$ measures the local angular rate of rotation in the fluid. In interactive analysis it has proven to be a useful feature for discriminating vortices of different strength.

The velocity gradient tensor $J=\nabla \mathbf{v}$ is defined as the Jacobian of $\mathbf{v}$. It describes the rate of change of the velocity along the principal directions and is computed in an intermediate step for most of the detectors.

The rate-of-strain tensor $S=\frac{1}{2}\left(\nabla \mathbf{v}+\nabla \mathbf{v}^{T}\right)$ is the symmetric part of the velocity gradient, and measures the deformation of a fluid cell element.

The rate-of-rotation tensor $\Omega=\frac{1}{2}\left(\nabla \mathbf{v}-\nabla \mathbf{v}^{T}\right)$ is the antisymmetric part of the Jacobian and measures the rotational movement of a fluid cell element.

A local coordinate system uses a frame of reference that 


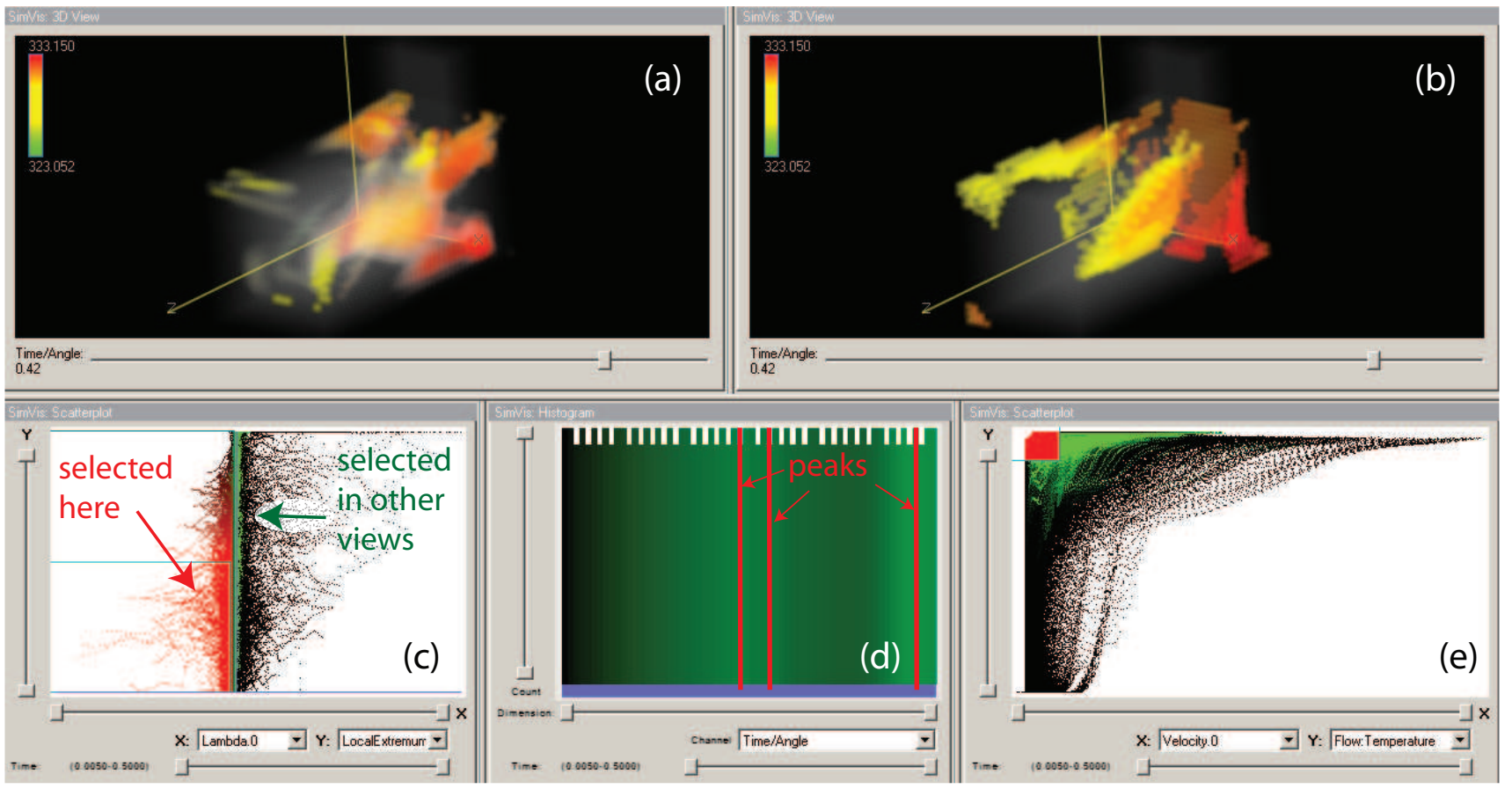

Figure 1: Multiple views and smooth brushing for vortex analysis. (a) A 3D view showing regions of high local vortexmeasures. (b) A 3D-view with low velocity and high temperature in focus serves as reference. (c) A scatterplot of the $\lambda_{2}$ vortex detector output vs. local extrema of $\lambda_{2}$. (d) The histogram shows the development of the features selected in the scatterplots and reveals three peaks of turbulence over time. (e) A scatterplot of velocity vs. temperature allows to compare attribute values of the selection (red) with those of the vortex regions (green).

is tuned to the local situation at a fluid element. The parameters of a local curvilinear coordinate system can give insight into the swirling motion of the fluid, $\nabla \mathbf{v}$ becomes

$$
\nabla \mathbf{v}=\left[\mathbf{v}_{\mathbf{r}} \mathbf{v}_{\mathbf{c r}} \mathbf{v}_{\mathbf{c i}}\right]\left(\begin{array}{ccc}
\lambda_{r} & & \\
& \lambda_{c r} & \lambda_{c i} \\
& -\lambda_{c i} & \lambda_{c r}
\end{array}\right)\left[\mathbf{v}_{\mathbf{r}} \mathbf{v}_{\mathbf{c r}} \mathbf{v}_{\mathbf{c i}}\right]^{-1}
$$

With $v_{r}, v_{c r}$ and $v_{c i}$ being eigenvectors of $J, \lambda_{r}$ the real eigenvalue and $\lambda_{c r} \pm i \lambda_{c i}$ the conjugated complex eigenvalue pair.

In their standard definition the common feature detectors give a binary classification for each volume element to the classes 'vortex' and 'no vortex'. In our work we have experienced that it is more useful to consider the whole spectrum of local feature detector response instead of using a binary threshold. Especially in combination with direct volume rendering more information is communicated by also showing the gradient information in the visualization. Nevertheless, the interaction framework still allows us to use thresholding and binary brushes.

\subsection{Feature Detectors}

Some of these feature detectors use the same building bricks as others but calculate the final result in a differ- ent way. Especially the parallel vectors operator [22] and the critical point criterion of Chong et al. [5] are common "building parts" for feature detection algorithms. For example by combining the two we get the detector introduced by Sujudi and Haimes [25]. This shows how multiple views and brushing can widen the possibilities of interactive analysis: by implementing the building bricks the user can combine these in standard ways using default values or combine them in new ways when the standard methods do not give satisfying results.

Vorticity magnitude The straightforward way to look for vortices is to search for regions of high vorticity magnitude. Vorticity magnitude thresholding is often used as a detection method to mark cells as potential vortex regions and apply local verification using computationally more demanding methods.

Helicity and Curl Levy et al. propose the use of normalized helicity and curl and search for regions where $v \| \omega[16]$. Even though this may not always correspond to the actual vortex core line, the authors used this feature with corresponding colors successfully on meteorological data.

Hunt's Q criterion compares the symmetric $(S)$ and the ro- 


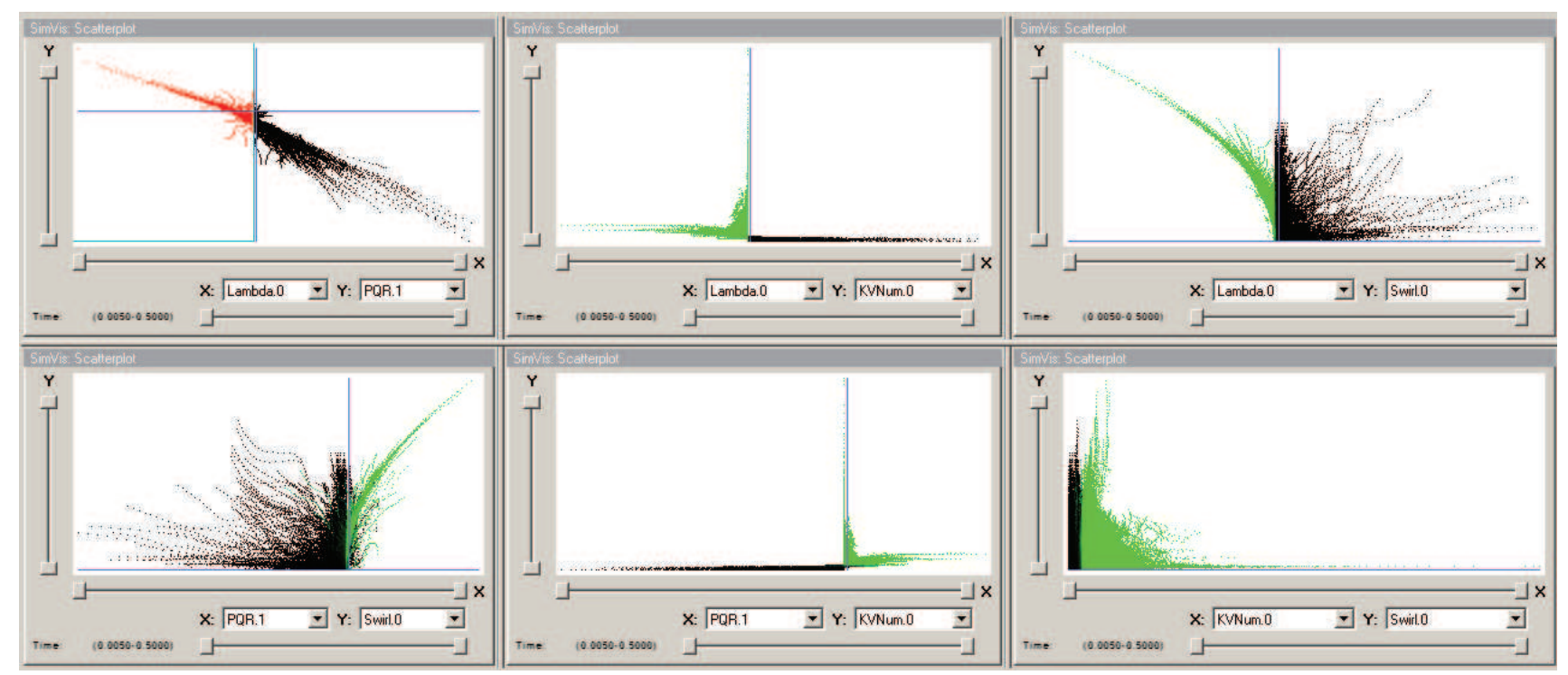

Figure 2: Combining multiple detectors using linked views: (a) Lambda vs. Q shows that the two are closely related in this data set. (b) The relation between $\lambda_{2}$ and kinematic vorticity number is smaller but the sharp 'edge' in the distribution helps to specify a good threshold for $\lambda_{2}$. (c) The comparison with the swirl criterion verifies that we have selected a good threshold. (d-e) the pairwise relations of $\mathrm{Q}$, the swirl criterion and the kinematic vorticity number can be used as a sanity check for the detected region.

tational component $(\Omega)$ of $J[10]$. Hunt combines this with the additional requirement of a local pressure minimum. In our framework we do not need to do this, since this additional requirement can be added interactively by brushing a derived quantity that finds local pressure minima.

Lambda2 introduced by Jeong and Hussain [12] is one of the most popular vortex region detectors and has been studied extensively over the years. The criterion involves computing the symmetric matrix $S^{2}+\Omega^{2}$ and its eigenvalues $\lambda_{1} \geq \lambda_{2} \geq \lambda_{3}$. A vortex is detected in the connected region where $\lambda_{2}$ is negative.

Kinematic Vorticity Number $N_{k}$ introduced by Truesdell [27] is a local measure that gives the quality of rotation independent from vorticity magnitude. Very large values correspond to solid body rotation and very small values to irrotational motion.

Chong's criterion is based on critical point theory and the eigenvalues and eigenvectors of the Jacobian. A material particle is considered to show spiraling motion if $\nabla v$ has two complex eigenvectors [5]. Perry and Chong give a description of the relation between the Jacobian and its invariants $P, Q$ and $R$ [18].

The swirling strength parameter of Berdahl [2] and related methods [30, 4] derive values measuring swirling from the imaginary and real parts of the complex eigenvalue pair. In interactive analysis these methods are useful since they give information on the local strength of swirling motion. To combine different vortex detectors using multiple views we have extended them to output smooth fuzzy response values [3].

In Figure 2 we see that the different detectors have unique response characteristics and inspection of their interaction can help visual analysis. Since the detectors are all based on values computed from the Jacobian there is very often some correspondence. Chakraborty et al. [4] present an analysis of the relations between local feature detectors. Nevertheless, the different criteria give different perspectives on the vortex features inside the dataset. Since the nature of vortex features is not fully understood, it is still necessary to experiment how to combine the different detectors especially to achieve confidence in the precise shape of feature boundaries. The fuzzyness of these boundaries makes it difficult to use a single detector and a single view to understand them.

\section{PQR Plots}

The analysis of critical points of the fluid field has proven to be very useful for understanding the behavior of flow fields. Perry and Chong discuss how critical point concepts can help understand eddying and swirling flow patterns $[18,5]$. They use three invariants of the Jacobian $P$, 


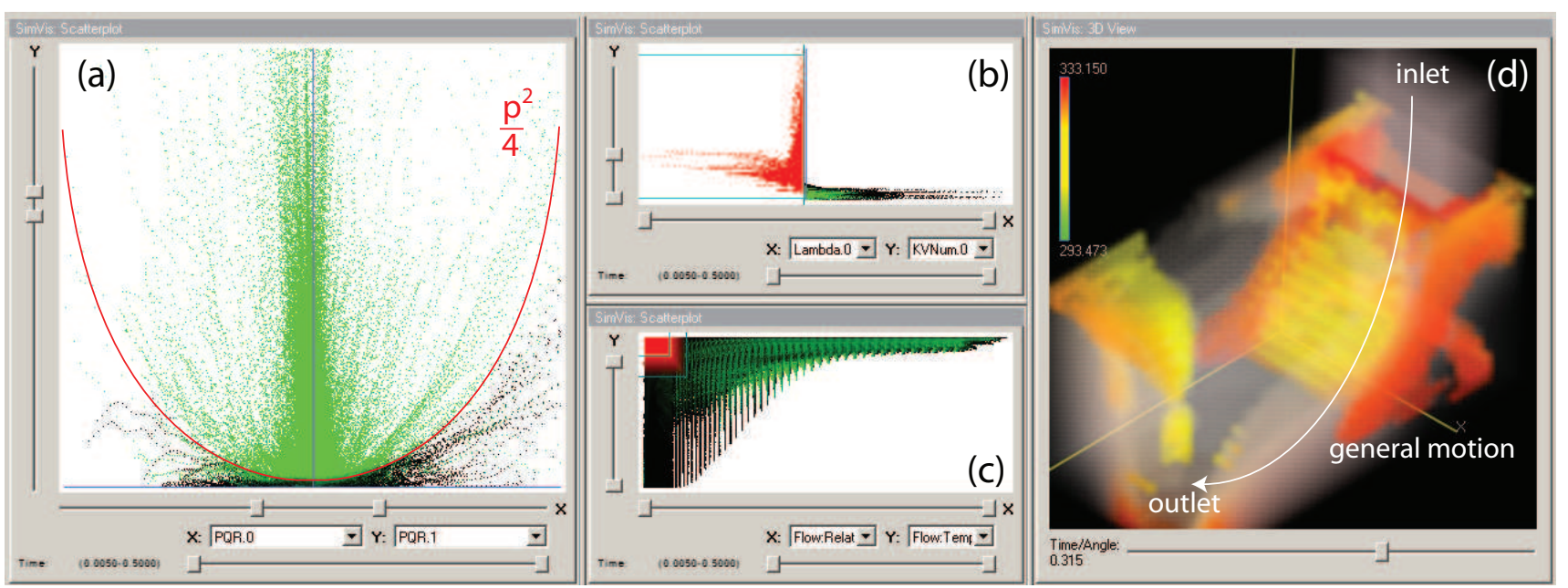

Figure 3: A PQ-plot: (a) most of the cells selected by the the OR-combination of the $\lambda_{2}$ method and the low-pressure-hightemperature feature lie above the parabola $q=\frac{p^{2}}{4}$ in the pq-plot (drawn red over the scatterplot for illustration). (b) The first feature (red) selects $\lambda_{2}$ values suggesting a vortex region. We see where this differs from the second feature (green). (c) The second feature (red) selects low pressure and high temperature. We see that most of the cells belonging to a vortex unexpectedly exhibit high pressure values (green). (d) In the 3D-view we can see the region in space where we have both features present.

$\mathrm{Q}$ and $\mathrm{R}$ :

$$
\begin{gathered}
P=-\operatorname{trace}(J), \quad Q=\frac{1}{2}\left(P^{2}-\operatorname{trace}\left(J^{2}\right)\right) \\
R=-\operatorname{det}(J)
\end{gathered}
$$

The invariants define the topology of possible patterns and tell about physical properties of the flow. Therefore they give a useful complement to the other views. Since plots of $P, Q$ and $R$ are traditionally used for presentation and discussion in the literature and not as vortex extraction criteria we do not scale or transform them in any way but map them directly to scatterplots. Plotting different planes of these invariants is a common tool when discussing flow topology features. Typically one invariant is held constant (e.g., $P$ vs. $Q$ with $R=0$ ) such that the result can be visualized using a 2D plot, but to our knowledge this has not been done interactively using multiple linked views, where it is possible to compare the distribution of points interactively for different settings. The invariants are related to the eigenvalues of the characteristic equation by

$$
\operatorname{det}(A-\lambda I)=0 \leftrightarrow \lambda^{3}+P \lambda^{2}+Q \lambda+R=0
$$

plots of $P, Q$ and $R$ give insight into the complex and real eigenvalues of the characteristic equation and therefore into parts of the flow which show eddying motion.

In Figure 3 the PQ-plot shows the spread of cells in the PQ-plane with $\mathrm{R}$ selected to be zero and compares this with the results of the $\lambda_{2}$ method. In (a) we see that most of the cells selected by the $\lambda_{2}$ method and the low-pressure-hightemperature feature lie inside above the parabola $q=\frac{p^{2}}{4}$ in the PQ-plot. This is the region of stable and unstable focus flow, that is a pattern of spiralling motion. In (b) the selection (red) is centered at high $\lambda_{2}$ values. Through linking we see where this differs from the second feature (green). (c) The selected feature (red) of low pressure and high temperature can be compared with the feature from the other views (i.e., b). We see that most of the cells belonging to a vortex exhibit high pressure values (green). This is an atypical finding, since due to the forces outwards the center of spiraling motion we commonly find lower pressure values connected with vortices. The $3 \mathrm{D}$ view (d) complements the analysis by showing the regions in space where we have at least one of the features present. The regions match with what would have been expected, that is to say behind the inlet, at the region on top opposite of the outlet and around the outlet.

\section{Correlation Brushing}

When trying to understand how several detectors combine we would like to understand how the response values of two detectors correlate. For this purpose we use a rotation of the scatterplot to be able to use rectangular brushing to find correlating parts of a feature. We calculate the offset and slope of the regression line and rotate the scatterplot to match the regression line with the $\mathrm{x}$-Axis. See Figure 4 $(\mathrm{e}+\mathrm{f})$ for an example. Let $X$ and $Y$ be the attributes under inspection in the scatterplot. Then we can compute the 

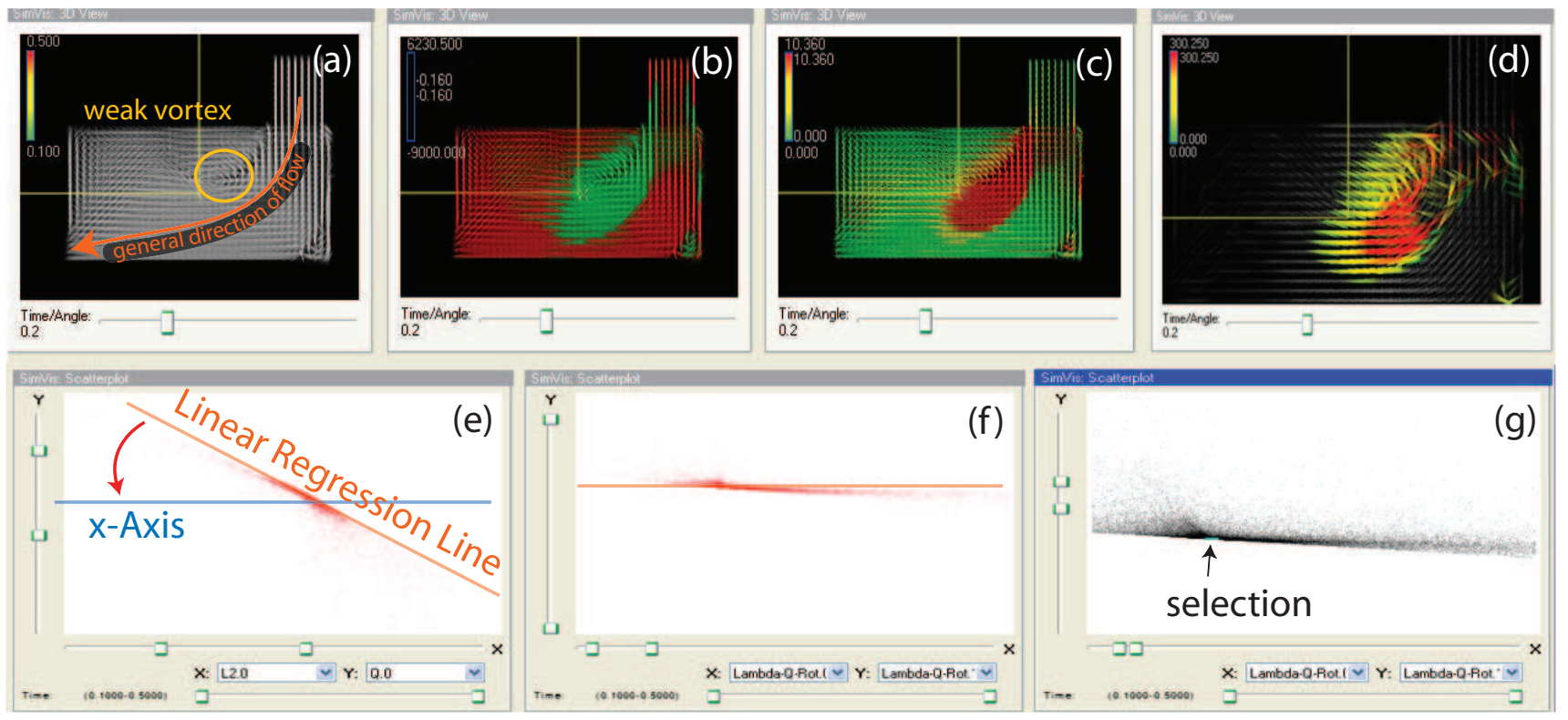

Figure 4: Correlating several detectors: (a) The dataset contains a weak and small vortex in a slow portion of the fluid, that no single method can find satisfyingly. (b) The $\lambda_{2}$ method has to be set to very high sensitivity to include the vortex region. (c) The swirling strength method detects a similar region. (d) The region selected by using a combined detector that correlates $\lambda_{2}$ and swirling strength. (e) The rotation of the points (see text) in the scatterplot is dependent on the slope of the regression line. (f) After rotation the regression line matches with the x-Axis. (g) A rectangular selection now contains an inherent measure of correlation of the detectors.

mean values $\bar{x} \bar{y}$ to translate the points in the plot such that $(\bar{x}, \bar{y})$ coincides with the origin of the scatterplot. The slope $\beta$ of the regression line $y=\alpha+\beta x,(\alpha=0$ after translation) is then

$$
\beta=\frac{\sum_{i}\left(\left(x_{i}-\bar{x}\right)\left(y_{i}-\bar{y}\right)\right)}{\sum_{i}\left(x_{i}-\bar{x}\right)^{2}}
$$

This gives the angle between the $\mathrm{x}$-Axis and the regression line as $\arctan (\beta)$. After rotating the points in the scatterplot we can use rectangular brushing to select correlating parts of the attributes $X$ and $Y$. Since linear regression using a regression line based on least-squares-fitting is very sensitive to outliers in the data, we include the option to exclude a certain percentage of the data from the calculation of the covariance (e.g. the 100th percentile) in the user interface.

In Figure 4 we see an example of this approach. The 3D images (a-c) are linked in relation to zoom and viewing parameters, while the fourth 3D view (d) has a linked projection parameter but larger zoom for closeup visualization. The scatterplot (g) and the 3D view (d) are linked such that the selected cells get full opacity. We study a weak vortex in a region behind the inlet where the flow is moving slowly close to a stronger main current from inlet to outlet. For this reason the general direction of the flow from inlet to outlet is difficult to distinguish from the location of the vortex by local feature detectors. In (b) and (c) we can see that both detectors tend to include some portion of the bend flow into the detected vortex region. Still, the strengths of response differ in a systematic way from each other: in the faster portion of the flow we get detector responses that correlate differently than the responses for fluid cells inside the weak vortex. Therefore we can select a good approximation to the vortex region with a single brush. Of course we could have improved the selected region by using additional brushes and including other features, but in the image we can clearly see that in the rotated view a single selection already performs very well.

\section{Multiple detector views}

Numerical data visualization aims at supporting the tasks of exploration and analysis to find out about certain characteristics of the dataset. Important tasks are: verification of simulation results by the experts for sanity, improvement and optimization of shape and geometry, definition of settings and parameters of controlling hardware (e.g., the chip controlling injection speed for different engine loads) and prediction of fluid-material interaction (e.g., transport of lubrication fluid). All these properties of the data are connected to a certain extent to the control of vortices and their development over time. 


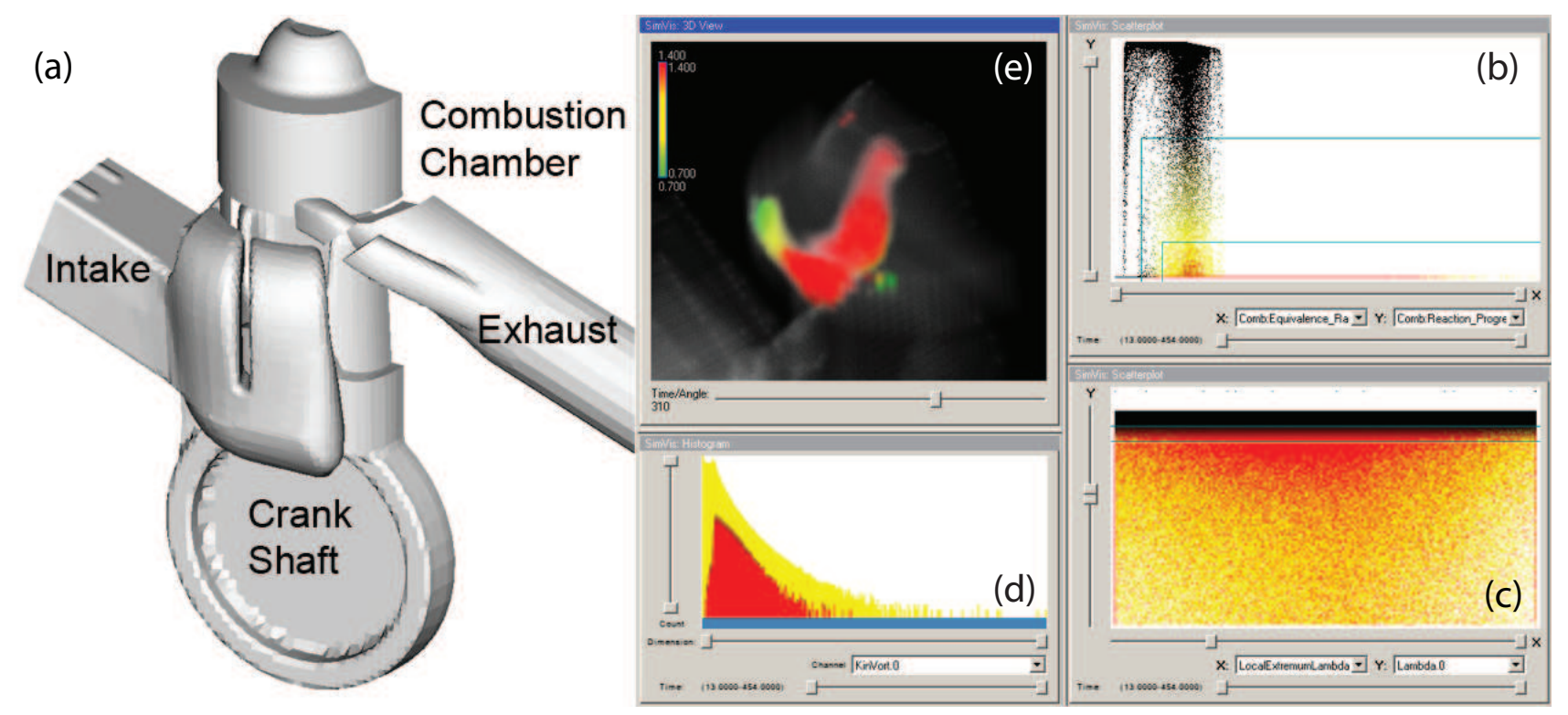

Figure 5: Feature localization analysis in the application case of a 2-stroke engine simulation: (a) an overview showing the engine geometry. (b) In the first step we select the parts of the data with the desired oxygen/fuel ratio. (c) To find out which portions of the vortex result in optimal mixing we plot $\lambda_{2}$ against local extremes of $\lambda_{2}$, the optimal mixing seems to result from medium vortex strength. (d) We check this result by comparing with a histogram of the kinetic vorticity number and see that the cells under strong rotation do not show optimal mixing ratios. (e) The resulting mixing vortex region colored by ER attribute ('ER" measures combustibility).

We have identified four important interaction schemes that are often used in interactive analysis - they all involve the use of multiple linked views:

- vortex and feature localization answers questions on the location of vortices and related features inside the data. The engineer uses interactive brushing in multiple views to select ranges of different features that are of interest. Different 3D views will show where the concerned regions are located and the user can relate regions and their properties with each other

For example in a simulation of two mixing fluids the user can search for regions of turbulent flow by brushing high vorticity values and compare them with regions of high concentrations of one fluid to find stable vortices that are interfering with the mixing process. (See Figure 5 for an example and its discussion later in the text.)

- spatial analysis answers questions concerning the properties of a certain subvolume of the data. The engineer selects the relevant region using projections and $3 \mathrm{D}$ views and uses multiple scatterplots to visualize the situation and compare it to the overall behavior of the fluid in other views. This kind of anal- ysis is necessary to understand the situation at important parts of the geometry to find out if the flow behaves as expected. See Figure 6 for an example.

- vortex-feature analysis answers questions on the relationships between vortices and other features. The engineer uses multiple connected views to gain understanding on how a selected feature effects other attributes of the data. For example the user can use several feature detectors to detect all vortices and to exclude false positives. Afterwards the engineer can analyze how the temperature in these regions compares to the overall situation. See Figure 7 for an example.

- time-dependent vortex analysis answers questions on the progression of properties of the data in time. The engineer uses multiple views that show the situation at different time steps or the development of a feature over time. For example the user can select a vortex and analyze its progression in the course of time.

The key to these analysis guides is the linking between the different views. When we need to interpret data - that is to go from numbers to meaning - we need the builtin connection between the different views: linked view- 

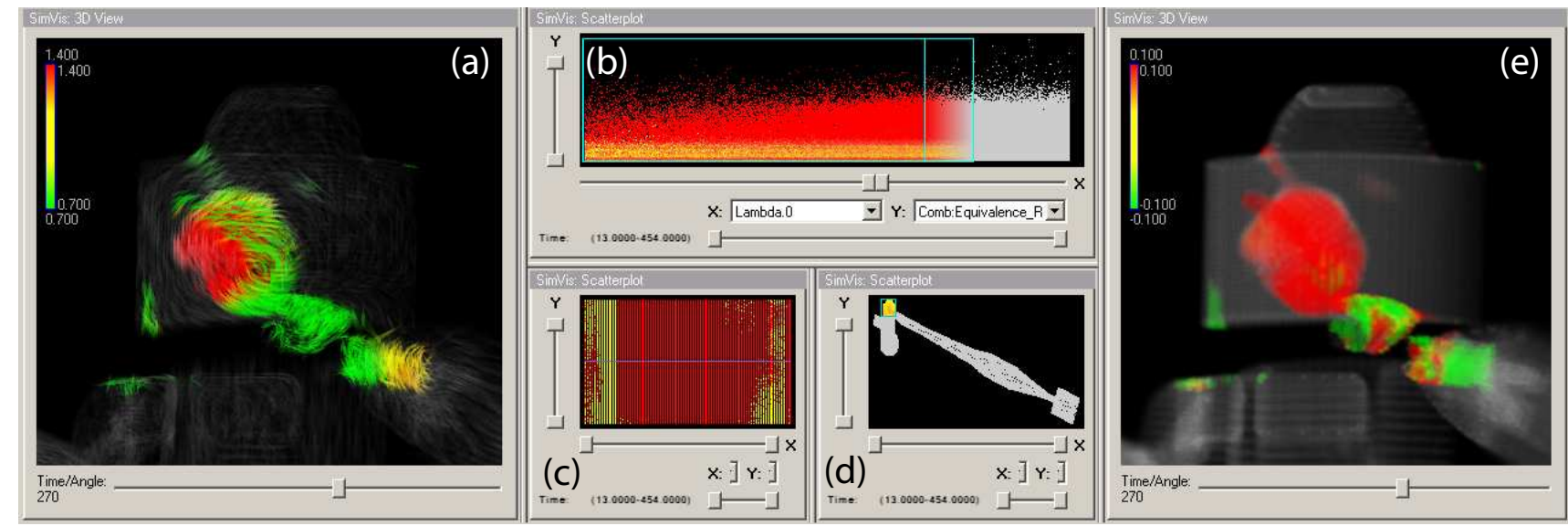

Figure 6: Analysis of the tumbling motion inside the combustion chamber: (a) a 3D view of the tumbling motion that causes mixing of fuel and oxygen. (b) The selection of high turbulence measure. (c) A scatterplot showing the distribution of time vs. helicity shows that the current timestep is of importance for the overall process. (d) A 2D projection of the engine were the combustion chamber is selected for spatial analysis. (e) A second 3D view with color mapped to helicity shows that the selected vortex consists of at least two counterrotating parts.

ing parameters in the 3D views during feature localization and linked feature brushing for inter-feature analysis and spatial analysis. This procedural approach to interactive drill-down analysis is based on a combination of computational, automatic feature detectors that are computed in a pre-processing step with interactive brushing and attribute selection.

We see that using multiple linked views and complementary feature detectors we can gain deepened insight of the vortex-type features inside the flow. The analysis involving several feature detectors gives two opportunities for further inspection. The first way is to interrelate different detectors to

- increase confidence in the detected region or core [26].

- find false positives and false negatives by comparing where the detectors agree and disagree.

- limit the response to a certain type of vortex feature. For example this can be beneficial to be able to discern vortices corresponding to the direction of rotation or strength of rotation.

These properties are important when dealing with a large number of automatically detected vortices, where it is necessary to analyze only the largest ones or otherwise classified as 'critical'. The combination of detectors and attributes of the data is beneficial to

- find vortices that are critical in the context. For example vortices that hinder mixing, that stall the fluid motion, take away kinetic energy or cause material wearout.

- analyze vortices that are caused by purpose to check their effectiveness and general shape (e.g., to cause mixing).

- understand how the existence of vortices is related to relevant properties inside the fluid.

All these tasks inevitably involve the use of multiple views, both $2 \mathrm{D}$ and $3 \mathrm{D}$, and require to relate features and detectors to each other.

\section{Application study}

Datasets from computational fluid dynamics (CFD) simulation share specific characteristics: they cover multiple aspects of the simulation related to physical properties of the material (e.g., temperature, density or strain), the fluid component (e.g., direction, kinetic energy or pressure) and additional quantities related to the application domain (e.g., rate of combustion or soot density). In the following we will discuss two examples of interactive visual analysis of simulation data from the field of automotive research.

\subsection{Two-Stroke Engine}

The 2-stroke engine dataset contains a complete simulation of the injection and combustion of fuel during one crank revolution. The model is based on a moving volume mesh such that at every time step a new unstructured grid is introduced. In a previous work, Schmidt et al. have analyzed this dataset with the SimVis approach [23] and compared the results with a VR/AR method. With the use 

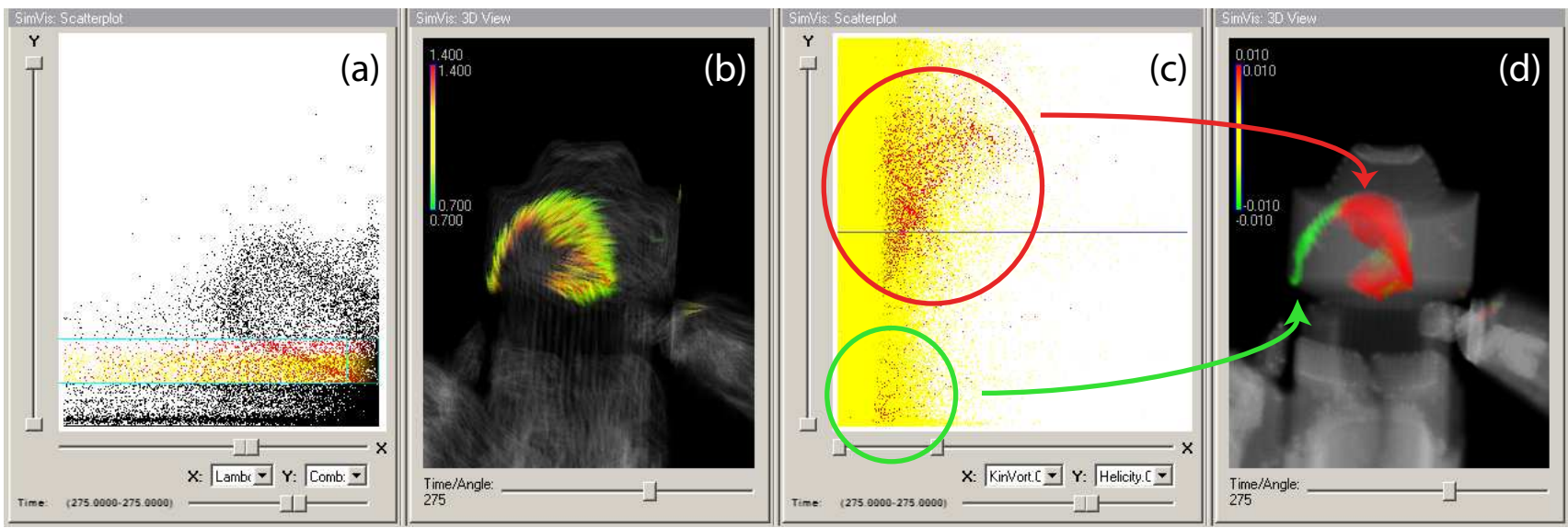

Figure 7: Further investigation of optimal mixing. (a) The portion of the vortex from Figure 6 where the mixing attribute lies inside the optimal range. (b) In the 3D view we can see that this region is located at the boundary of the rotating motion. (c) Plotting kinematic vorticity vs. helicity reveals two clusters with a tendency to slowly rotating motion. (d) In 3D we can see that the region of optimal mixing differs from the main parts of the tumbling motion (compare Fig. 6 (e)).

of CFD simulation initial experiments can be made without costly production of prototype hardware. In this step, unexpected processes can be investigated and the course of physical processes can be shaped according to the engine specifications. One key attribute that is related both to emission and engine performance is equivalence ratio (ER), which is the relation between fuel and air within a volume cell. It is crucial that ER lies in the optimal interval between 0.7 and 1.4 for most fluid cells at the moment of ignition. In Figure 5 we inspect the mixing process inside the combustion chamber - using the approach following a spatial feature analysis process: (b) in the first step we see the region of optimal mixing in a timestep at the beginning of the compression process when the reaction has not started yet. (c) This region was selected using a scatterplot showing ER and a reaction progression attribute. (d) A scatterplot that relates vortex detector output against its local extrema shows that optimal mixing is related to regions that are not at the peaks of vortex detector response. This could be related to the ambivalent nature of vortices in relation to mixing: a strong vortex can isolate portions of the flow inside and hinder mixing while chaotic motion and turbulence tend to cause full mixing of different fluid materials. (e) The histogram of kinematic vorticity shows clearly that the parts of the fluid that have reached optimal mixing (colored red in the histogram) are rotating slower than the rest.

In order to further analyze the connection between turbulent motion and mixing inside the combustion chamber we continue with a spatial analysis. In Figure 6 a measure of high turbulence is selected and related to the region that exhibits optimal mixing. In (a) we see a visualization of the rotating motion that is part of the tumbling motion which is the main force of the mixing process of fluid and oxygen. This first orientation step of the spatial analysis shows the vortex in nearly full development. This vortex constitutes an important part of turbulent motion inside the combustion chamber and is essential in the mixing process during compression resulting from the upward motion of the piston. Even though we have mapped color to ER, spatial relations do not become immediately clear. (b) In the scatterplot we can see that ER in the combustion chamber is contained in a very narrow band (the yellow portion) probably due to the effect of the injection setup. We have selected high vortex-measure $\left(\lambda_{2}\right)$ values. (c) A scatterplot of time vs. helicity shows a tendency to clockwise rotation in later timesteps. (d) We have used a $2 \mathrm{D}$ projection of the geometry to select the combustion chamber for local analysis. (e) Mapping helicity to color of the volume cells we can distinguish the main vortex (red) from a smaller turbulent region (green).

To understand the properties of this vortex we continue with an inter-feature analysis that relates features of the fluid with ER. This is shown in Figure 7. (a) As a first step we select optimal ER in a scatterplot showing the situation in the current timestep. (b) The 3D view shows that the portion of the vortex where the mixing attribute lies inside the optimal range is located at the boundary of the vortex. (c) Plotting kinematic vorticity vs. helicity reveals two clusters, both with a tendency to slower rotating motion. (d) In a $3 \mathrm{D}$ view we can see that the region of optimal mixing differs from the main parts of the tumbling motion (compare Fig. 6 (e)). We can assume that the second cluster (green) is a weaker child vortex caused by the main vortex and that 


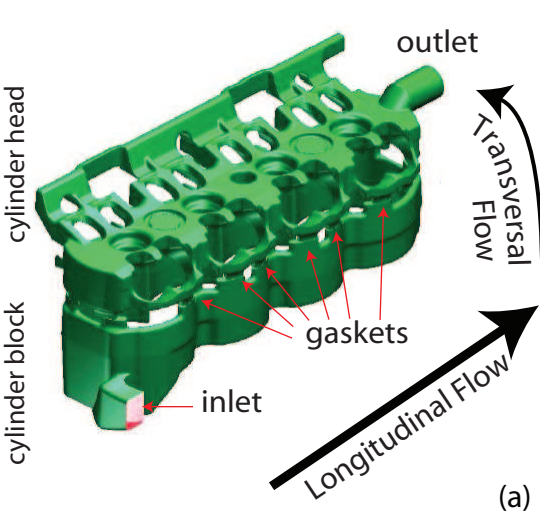

(a)
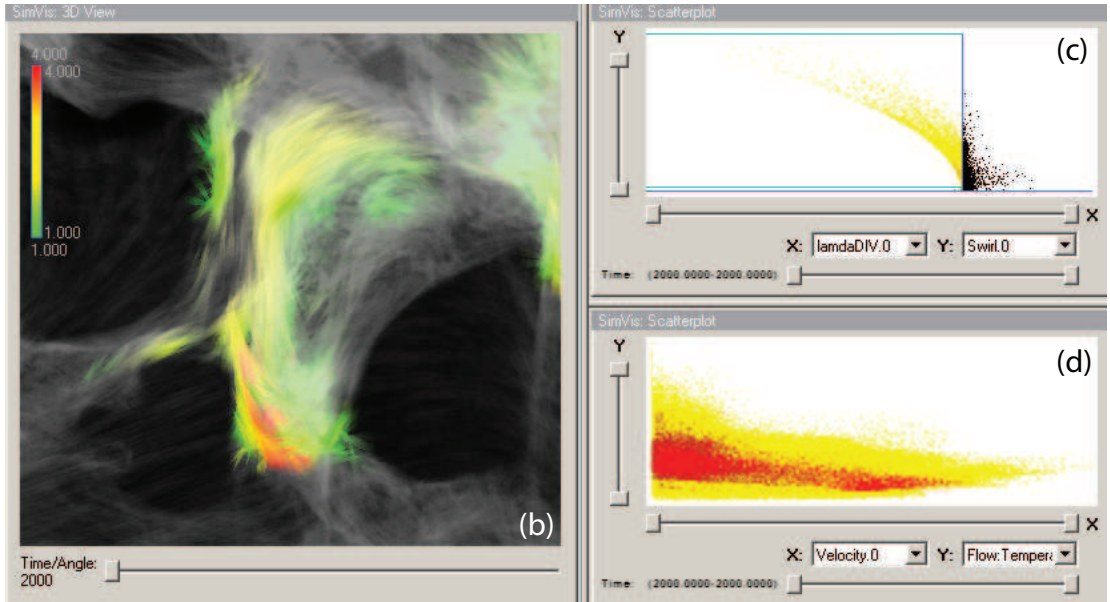

Figure 8: One key part of the flow inside the cooling jacket are the vortices caused by the gaskets between the cylinder block and cylinder head parts. (a) An overview of the cooling jacket geometry [3]. (b) A closeup of one of the vortices behind a gasket (see also Fig. 9 (a) no. 1). (c) The central parts of the vortices can be selected by combining $\lambda_{2}$ and high swirl. (d) When plotting velocity against temperature the gasket vortices lie inside a single cluster.

the mixing process is related to the appearance of smaller regions of turbulence caused by the main tumbling motion.

\subsection{Cooling Jacket}

In the second application example we inspect fluid flow through a cooling jacket. In this application evaluation we continue work done by Garth et al. [8] and Laramee et al. [15] where the regions of turbulence behind the gaskets were not considered. Computational fluid dynamics software is used to inspect and improve the design process and we know that engineers invest large amounts of time to optimize the geometry of cooling jackets.

The cooling jacket in focus (see Figure 8 (a)) is designed for a four cylinder engine for cars. The shape of the cooling jacket is the result of multiple factors including the shape of the engine block and the optimal working temperature for the particular engine. The cooling jacket geometry consists mainly of three components: the cylinder head on top, the cylinder block on the bottom and a thin component connecting the cylinder head and block called the gasket. The cylinder head is responsible for transferring heat away from the intake and exhaust ports at the top of the engine block. The cylinder block is responsible for heat transfer from the engine cylinders and for even distribution of flow to the head. Between the cylinder head and block lies the cooling jacket gasket. It consists of a series of small holes that act as conduits between block and head. These ducts can be quite small relative to the overall geometry but they are nonetheless very important because they are used to govern the motion of fluid flow through the cooling jacket.

The coolant flow inside the jacket is governed by two major directions - a longitudinal motion along the cylinders of the engine and a transversal motion from cylinder block to head. To keep the engine operative it is necessary to obtain an even distribution of flow to each engine cylinder and to avoid regions of stagnant flow to ensure good overall heat transport.

Around the combustion chambers heat is transported away from the surface by a mixing process that replaces the heated fluid. To this end the shape of the gaskets causes turbulent regions behind the gaskets. The turbulent regions differ in size and heat transport efficiency. In Figure 8 (b) we see a closeup of a single vortex with color mapped to velocity. (c) We use a combination of $\lambda_{2}$ and the swirling strength parameter to select the full region of turbulence restricted to the parts rotating stronger. In (d) we see a scatterplot involving all vortices and so far we cannot distinguish differences between the two vortices in relation to their attributes.

In Figure 9 (a) we see six of these turbulent regions in focus with the rest of the geometry in shades of gray for context visualization. In scatterplots visualizing detector outputs ((d) and (e)) we cannot see obvious differences between the turbulent regions 1 and 2. Therefore we switch to analyzing their attribute values using additional linked views. In a scatterplot (b) of turbulent kinetic energy vs. velocity we see that the second vortex (red) tends towards slower movements and less kinetic energy. A second scatterplot (c) now reveals that the two turbulent regions differ in their temperature attributes. Further analysis shows that they are similar in other aspects. We can conclude that the difference between the two regions of turbulence stems 

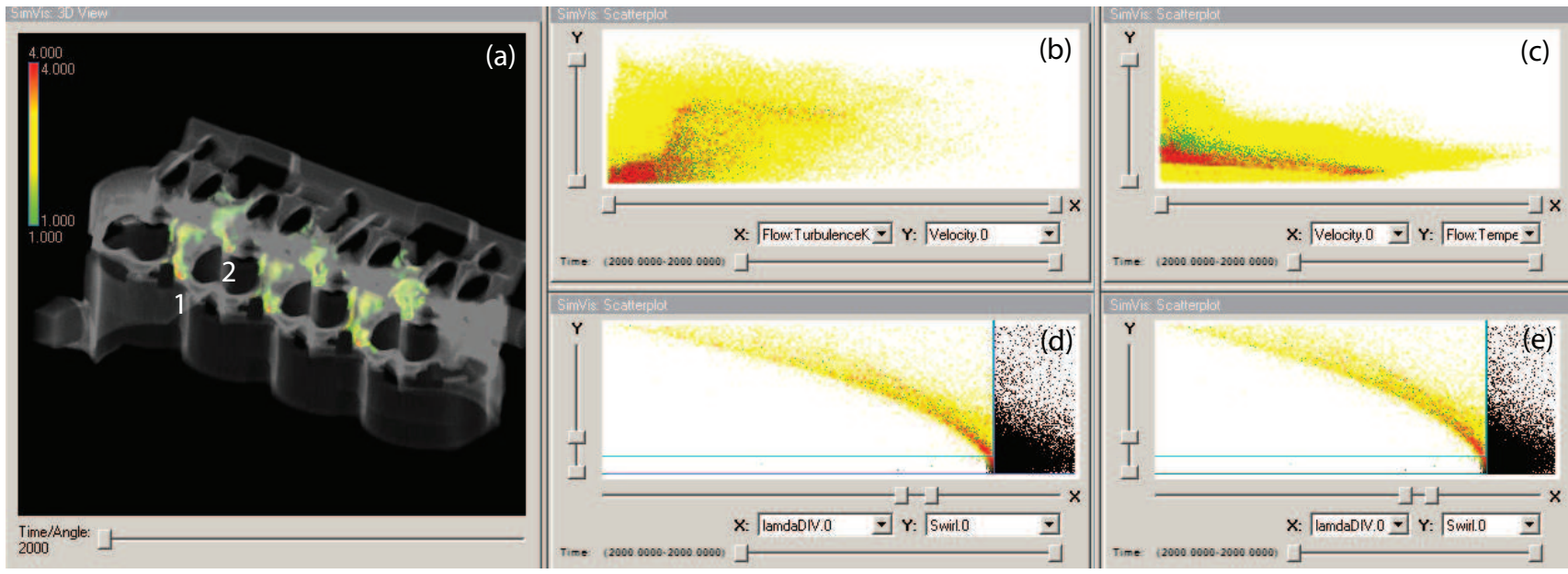

Figure 9: We analyze the difference between two of the vortices caused by the gasket geometry: (a) an overview of the gasket vortices in the cooling jacket geometry. (b) This scatterplot shows that second vortex (red) tends to lower turbulence and velocity but clear distinction is not possible. (c) We found the differing attribute: plotting temperature separates the two regions. $(\mathrm{d}+\mathrm{e})$ Both vortices are very similar in the outputs of the vortex detectors.

from the larger temperature of the fluid before entering gasket 2. This is due to the fact that the fluid arriving at the second gasket has covered a larger distance from the inlet than the fluid that goes through gasket 1 . The result is therefore that the slightly lower cooling effect of the second gasket is not due to the gasket geometry but an acceptable effect of the overall situation.

\section{Technical Considerations}

SimVis has to handle data sets with up to 1000 time steps, 20-50 data attributes and up to several million cells. Therefore it is important to save resources available when calculating derived features. In this respect storing the feature information that was output by the detector in a float channel is a very good solution since the computations have to be done only once. The additional amount of data is comparatively small and the lazy loading capability of the SimVis implementation supports this approach.

To achieve fast rendering speeds for the large amount of cells we use the vertex buffer object arrays to store the information necessary for rendering directly on graphics hardware. To further speed up computationally intensive processes, we use the SSE (Streaming SIMD Extensions) instruction set. This allows to perform calculations on four 32 bit float values simultaneously. It is possible to optimize the calculation of the degree of interest function and fuzzylogic operations based on this extension, since it packs four operations into one. The SimVis system currently runs interactively on a standard PC (AMD Athlon 64 Dual Core 2,2GHz with a NVidia GeForce 6800 graphics card).

\section{Conclusions}

We have presented a case study of vortex feature analysis using combined detectors and multiple views in the context of CFD data for automotive industries research. Using multiple views it is possible to compare and weight the response values of several detectors intuitively by means of interactive visual analysis. Furthermore we have presented an extension to brushing features in scatterplots that allows to analyze the correlation of features using rectangular brushing. It was demonstrated that by correlating two detectors one can find features that could not be studied before. The case study reveals that the combination of multiple 2D and 3D views can help understanding complex 3D flow features when linking and brushing are combined appropriately. We have identified systematic approaches to study vortex phenomena in multidimensional datasets, based on multiple views, linking and combined feature detectors.

In the context of an application study the benefits of multiple linked views become clear: taking advantage of the combined potential of infovis views, 3D graphics visualization and automated feature detectors allows to understand the situation inside the data. Reading between the lines and gaining deepened understanding is possible by the streamlined and integrated approach of coordinated views and multiple detectors. For supplementary documentation and results to this case study, in particular video, high-resolution images and other material, we refer to www.vrvis.at/via/research/ multi-views-case/. Future work in multiple coordinated views contains a generalized approach to linked 
brushing and navigation that allows to link several views in a generalized fashion such that the analysis process is assisted with adapting 3D presentation.

\section{Acknowledgements}

The authors thank Meister Eduard Gröller for his guidance. This work has been partly funded by the FWF PVG project supported by the Austrian Science Fund (FWF) under grant no. P18547-N04, as well as by the "Bridge" funding program of the Austrian Funding Agency (FFG) in the scope of the "MulSimVis" project (Nr. 812106). The 2-stroke engine dataset is courtesy of the "Institut für Verbrennungskraftmaschinen und Thermodynamik" at the Technical University of Graz. The cooling jacket CFD simulation dataset is courtesy of AVL List GmbH, Graz, Austria.

\section{References}

[1] M. Q. W. Baldonado, A. Woodruff, and A. Kuchinsky. Guidelines for using multiple views in information visualization. In AVI '00: Proceedings of the working conference on Advanced visual interfaces, pages 110-119, 2000.

[2] C. H. Berdahl and D. S. Thompson. Eduction of swirling structure using the velocity gradient tensor. AIAA Journal, 31(1):97-103, 1993.

[3] R. B"urger, P. Muigg, M. Ilcík, H. Doleisch, and H. Hauser. Integrating local feature detectors in the interactive visual analysis of flow simulation data. In Proceedings of Joint Eurographics - IEEE VGTC Symposium on Visualization 2007, pages 171-178, 2007.

[4] P. Chakraborty, S. Balachandarand, and R. J. Adrian. On the relationships between local vortex identification schemes. Journal of Fluid Mechanics, 535:189-214, 2005.

[5] M. S. Chong, A. E. Perry, and B. J. Cantwell. A general classification of three-dimensional flow fields. Physics of Fluids Archive, 2:765-777, 1990.

[6] P. A. Davidson. Turbulence: An Introduction for Scientists and Engineers. Oxford University Press, 2004.

[7] H. Doleisch, M. Gasser, and H. Hauser. Interactive feature specification for focus+context visualization of complex simulation data. In VisSym2003, pages $239-248$, 2003

[8] C. Garth, R. S. Laramee, X. Tricoche, and J. Schneider. Extraction and visualization of swirl and tumble motion from engine simulation data. In Proc. of the Topology-Based Methods in Visualization Workshop, 2005.

[9] R. Haimes and D. Kenwright. On the velocity gradient tensor and fluid feature extraction. In Proceedings of AIAA 14th Computational Fluid Dynamics Conference, 1999.

[10] J. C. R. Hunt, A. A. Wray, and P. Moin. Eddies, stream and convergence zones in turbulent flows. In 2. Proceedings of the 1988 Summer Program, pages 193-208, 1988.

[11] F. Hussain. Coherent structures - reality and myth. Phys. Fluids, 26:2816, 1983.
[12] J. Jeong and F. Hussain. On the identification of a vortex. Journal of Fluid Mechanics, 285:69-84, 1995.

[13] D. A. Keim. Information visualization and visual data mining. IEEE Transactions on Visualization and Computer Graphics, 8(1):1-8, 2002.

[14] L. Lam. Classifier combinations: Implementations and theoretical issues. In MCS '00: Proc. Workshop on Multiple Classifier Systems, pages 77-86, 2000.

[15] R. S. Laramee, C. Garth, H. Doleisch, J. Schneider, H. Hauser, and H. Hagen. Visual analysis and exploration of fluid flow in a cooling jacket. In Proceedings IEEE Visualization 2005, pages 623-630, 2005.

[16] Y. Levy, D. Degani, and A. Seginer. Graphical visualization of vortical flows by means of helicity. AIAA Journal, 28:1347-1352, 1990.

[17] H. Miura and S. Kida. Identification of tubular vortices in turbulence. Journal of the Physical Society of Japan, 66:1331-1334, 1997.

[18] A. Perry and M.S. Chong. A description of eddying motions and flow patterns using critical point concepts. Annual Review of Fluid Mechanics, 19:125-155, 1987.

[19] F. Post, B. Vrolijk, H. Hauser, R. Laramee, and H. Doleisch. The state of the art in flow visualization: Feature extraction and tracking. Computer Graphics Forum, 22(4)(2):775792, 2003.

[20] F. Reinders, H. Spoelder, and F. Post. Experiments on the accuracy of feature extraction. In Proc. 9th EG Workshop on Visualization in Scientific Computing '98, pages 49-58. Springer-Verlag, 1998.

[21] Fabio Roli and Josef Kittler. Fusion of multiple classifiers. Information Fusion, 3(4):243, 2002.

[22] M. Roth and R. Peikert. A higher-order method for finding vortex core lines. In Proceedings IEEE Visualization '98, pages 143-150, 1998.

[23] S. Schmidt et al. Novel visualisation and interaction techniques for gaining insight into fluid dynamics in internal combustion engines. In Proceedings of Nafems World Congress, 2005.

[24] B. Shneiderman. The eyes have it: A task by data type taxonomy for information visualizations. In $V L$ '96: Proceedings of the 1996 IEEE Symposium on Visual Languages, page 336, 1996.

[25] D. Sujudi and R. Haimes. Identification of swirling flow in 3D vector fields. Technical Report AIAA-95-1715, American Institute of Aeronautics and Astronautics, 1995.

[26] J. J. Thomas and K. A. Cook. A visual analytics agenda. IEEE Comput. Graph. Appl., 26(1):10-13, 2006.

[27] C. A. Truesdell. The Kinematics of Vorticity. Indiana University Science Serie 19, 1954.

[28] Webpage about the SimVis Visualization Framework. See URL: http://www.simvis.at//.

[29] P. C. Wong. Guest editor's introduction: Visual data mining. IEEE Computer Graphics and Applications, 19(5):20-21, 1999. 
[30] J. Zhou, S. Balachandar, and R.J. Adrian. Mechanisms for generating coherent packet of hairpin vortices in near- wall turbulence. Journal of Fluid Mechanics, 387:353-396, 1999. 